Supporting information

\title{
Chemical Fingerprinting of Conifer Needle Essential Oils and Solvent Extracts by Ultrahigh-Resolution Fourier Transform Ion Cyclotron Resonance Mass Spectrometry
}

\author{
Omolara O. Mofikoya, Marko Mäkinen, and Janne Jänis*
}

University of Eastern Finland, Department of Chemistry, P.O. Box 111, FI-80101 Joensuu, Finland

*Corresponding author: Email: janne.janis@uef.fi, Tel.+358-50-4601057

Contents:

Supporting Tables S1-S4

Supporting Figures S1 and S2 
Table S1. Compounds identified with (-) ESI from conifer needle essential oils.

\begin{tabular}{|c|c|c|c|c|c|c|c|c|c|}
\hline $\begin{array}{l}\text { Calculated } \\
m / z[\mathrm{M}-\mathrm{H}]^{-}\end{array}$ & $\begin{array}{c}\text { Observed } \\
m / z[\mathrm{M}-\mathrm{H}]^{-}\end{array}$ & $\begin{array}{l}\text { Error } \\
(\mathrm{ppm})\end{array}$ & DBE & $\begin{array}{c}\text { Elemental } \\
\text { formula }\end{array}$ & \multicolumn{4}{|c|}{ Relative intensity (\%) } & \multirow[t]{2}{*}{ Putative compound(s) } \\
\hline & & & & & Pine & Spruce & Larch & Juniper & \\
\hline 101.060803 & 101.060787 & 0.1583 & 1 & $\mathrm{C}_{5} \mathrm{H}_{10} \mathrm{O}_{2}$ & 0.2434 & N.D. & N.D. & N.D. & 3-Methylbutanoic acid \\
\hline 121.029503 & 121.029504 & 0.0103 & 5 & $\mathrm{C}_{7} \mathrm{H}_{6} \mathrm{O}_{2}$ & 1.1166 & 0.2880 & 0.6484 & 0.1846 & Benzoic acid \\
\hline 133.065888 & 133.065877 & 0.0827 & 5 & $\mathrm{C}_{9} \mathrm{H}_{10} \mathrm{O}_{1}$ & 0.0398 & N.D. & N.D. & N.D. & Cinnamyl alcohol \\
\hline 137.024418 & 137.024414 & 0.0292 & 5 & $\mathrm{C}_{7} \mathrm{H}_{6} \mathrm{O}_{3}$ & 0.0332 & 0.0327 & N.D. & N.D. & Salicyclic acid \\
\hline 143.107753 & 143.107756 & 0.0192 & 1 & $\mathrm{C}_{8} \mathrm{H}_{16} \mathrm{O}_{2}$ & 1.2584 & 0.8338 & 0.7156 & 1.2582 & Octanoic acid \\
\hline 147.045153 & 147.045165 & 0.0816 & 6 & $\mathrm{C}_{9} \mathrm{H}_{8} \mathrm{O}_{2}$ & 0.0212 & 0.0220 & N.D. & N.D. & Cinnamic acid \\
\hline 149.097189 & 149.097200 & 0.0740 & 4 & $\mathrm{C}_{10} \mathrm{H}_{14} \mathrm{O}_{1}$ & 0.0909 & 0.0422 & 0.1290 & 0.8362 & Verbenone \\
\hline 151.040068 & 151.040067 & 0.0588 & 5 & $\mathrm{C}_{8} \mathrm{H}_{8} \mathrm{O}_{3}$ & 0.1205 & 0.7678 & 0.0091 & N.D. & 4-hydroxyphenyl acetic acid \\
\hline 153.128489 & 153.128504 & 0.0980 & 2 & $\mathrm{C}_{10} \mathrm{H}_{18} \mathrm{O}_{1}$ & N.D. & N.D. & 0.0115 & N.D. & Terpinen-4-ol \\
\hline 163.076453 & 163.076463 & 0.0598 & 5 & $\mathrm{C}_{10} \mathrm{H}_{12} \mathrm{O}_{2}$ & 0.2556 & 0.1549 & 0.0173 & 0.1059 & Eugenol \\
\hline 165.055718 & 165.055718 & 0.0000 & 5 & $\mathrm{C}_{9} \mathrm{H}_{10} \mathrm{O}_{3}$ & 0.0504 & 0.0968 & N.D. & 0.0063 & Dihydro-p-coumaryl acid \\
\hline 167.034982 & 167.035005 & 0.1377 & 5 & $\mathrm{C}_{8} \mathrm{H}_{8} \mathrm{O}_{4}$ & 0.0262 & 0.0339 & N.D. & 0.0078 & Vanillin \\
\hline 167.107753 & 167.107764 & 0.0643 & 3 & $\mathrm{C}_{10} \mathrm{H}_{16} \mathrm{O}_{2}$ & 4.7921 & 5.8765 & 0.0333 & 3.0039 & Geranic acid \\
\hline 169.123403 & 169.123408 & 0.0296 & 2 & $\mathrm{C}_{10} \mathrm{H}_{18} \mathrm{O}_{2}$ & 21.6252 & 25.5989 & 0.0371 & 9.2987 & Citronellic acid \\
\hline 171.139053 & 171.139069 & 0.0920 & 1 & $\mathrm{C}_{10} \mathrm{H}_{20} \mathrm{O}_{2}$ & 1.9039 & 1.9187 & 0.5046 & 1.4933 & Decanoic acid \\
\hline 177.092103 & 177.092092 & 0.0635 & 5 & $\mathrm{C}_{11} \mathrm{H}_{14} \mathrm{O}_{2}$ & 0.0188 & 0.0263 & 0.0122 & 0.0305 & Methyl eugenol \\
\hline 181.123403 & 181.123410 & 0.0400 & 3 & $\mathrm{C}_{11} \mathrm{H}_{18} \mathrm{O}_{2}$ & 0.0623 & 0.1167 & 0.0116 & 0.0304 & Terpinyl formate \\
\hline 183.102668 & 183.102680 & 0.0065 & 3 & $\mathrm{C}_{10} \mathrm{H}_{16} \mathrm{O}_{3}$ & 0.1679 & 0.1296 & 0.0489 & 0.0867 & Pinonic acid \\
\hline 183.139053 & 183.139072 & 0.1065 & 2 & $\mathrm{C}_{11} \mathrm{H}_{20} \mathrm{O}_{2}$ & 0.4203 & 0.5523 & 0.1860 & 0.2925 & Z-3-Hexenyl isovalerate \\
\hline 185.081932 & 185.081953 & 0.1121 & 3 & $\mathrm{C}_{9} \mathrm{H}_{14} \mathrm{O}_{4}$ & 0.1602 & 0.0686 & 0.0647 & 0.1472 & Pinic acid \\
\hline 191.056112 & 191.056153 & 0.2146 & 2 & $\mathrm{C}_{7} \mathrm{H}_{12} \mathrm{O}_{6}$ & 0.0308 & 0.0761 & 0.0110 & 0.0324 & Quinic acid \\
\hline 193.050632 & 193.050629 & 0.0173 & 6 & $\mathrm{C}_{10} \mathrm{H}_{10} \mathrm{O}_{4}$ & 0.0193 & 0.0417 & N.D. & 0.0075 & Ferulic acid \\
\hline 193.123403 & 193.123431 & 0.1450 & 4 & $\mathrm{C}_{12} \mathrm{H}_{18} \mathrm{O}_{2}$ & 0.0485 & 0.0757 & 0.0731 & 0.0548 & Myrtenyl acetate \\
\hline 195.139053 & 195.139064 & 0.0577 & 3 & $\mathrm{C}_{12} \mathrm{H}_{20} \mathrm{O}_{2}$ & 0.1248 & 0.1848 & 0.0486 & 4.5168 & Terpinyl acetate \\
\hline 205.123403 & 205.123430 & 0.1292 & 5 & $\mathrm{C}_{13} \mathrm{H}_{18} \mathrm{O}_{2}$ & 0.0539 & 0.2114 & 0.0103 & N.D. & Phenylethyl-3-methyl butanoate \\
\hline 205.159789 & 205.159796 & 0.0341 & 4 & $\mathrm{C}_{14} \mathrm{H}_{22} \mathrm{O}_{1}$ & 0.0633 & N.D. & 0.0755 & 0.2079 & $\alpha$-Irone \\
\hline 209.081932 & 209.081942 & 0.0454 & 5 & $\mathrm{C}_{11} \mathrm{H}_{14} \mathrm{O}_{4}$ & 0.0317 & 0.0364 & 0.0263 & N.D. & Sinapyl alcohol \\
\hline 209.154703 & 209.154714 & 0.0526 & 3 & $\mathrm{C}_{13} \mathrm{H}_{22} \mathrm{O}_{2}$ & 0.1467 & 0.1999 & 0.2829 & 0.1291 & Isobornyl propionate \\
\hline 215.165268 & 215.165286 & 0.0837 & 1 & $\mathrm{C}_{12} \mathrm{H}_{24} \mathrm{O}_{3}$ & N.D. & N.D. & 0.1071 & 0.0374 & Sabinic acid \\
\hline 215.165268 & 217.159825 & 0.1658 & 5 & $\mathrm{C}_{15} \mathrm{H}_{22} \mathrm{O}_{1}$ & 0.0194 & N.D. & 0.0106 & 0.0448 & Nootkatone \\
\hline 219.175439 & 219.175448 & 0.0395 & 4 & $\mathrm{C}_{15} \mathrm{H}_{24} \mathrm{O}_{1}$ & 0.1145 & 0.0235 & 0.0116 & 0.1006 & Caryophyllene oxide \\
\hline 221.081932 & 221.081946 & 0.0645 & 6 & $\mathrm{C}_{12} \mathrm{H}_{14} \mathrm{O}_{4}$ & 0.0466 & 0.0233 & 0.1101 & 0.0280 & Dillapiole \\
\hline 221.191089 & 221.191093 & 0.0158 & 3 & $\mathrm{C}_{15} \mathrm{H}_{26} \mathrm{O}_{1}$ & 0.0545 & N.D. & 0.3763 & 0.0570 & Germacrene D-4-ol \\
\hline 227.071368 & 227.071389 & 0.0910 & 9 & $\mathrm{C}_{14} \mathrm{H}_{12} \mathrm{O}_{3}$ & 0.5709 & 0.0669 & N.D. & 0.0246 & Benzyl salicylate \\
\hline 227.201654 & 227.201674 & 0.0866 & 1 & $\mathrm{C}_{14} \mathrm{H}_{28} \mathrm{O}_{2}$ & 5.8457 & 7.8471 & 6.7531 & 11.5091 & Ethyl laurate \\
\hline 237.186004 & 237.186029 & 0.1043 & 3 & $\mathrm{C}_{15} \mathrm{H}_{26} \mathrm{O}_{2}$ & 0.4452 & 0.2997 & 0.0469 & 0.2204 & Geranyl isovalerate \\
\hline 239.201654 & 239.201685 & 0.1275 & 2 & $\mathrm{C}_{15} \mathrm{H}_{28} \mathrm{O}_{2}$ & 0.3200 & 0.6206 & 0.5442 & 0.2858 & Methyl isovalerate \\
\hline 253.217304 & 253.217330 & 0.1007 & 2 & $\mathrm{C}_{16} \mathrm{H}_{30} \mathrm{O}_{2}$ & 1.0361 & 1.5414 & 2.8826 & 1.1305 & Cyclohexadecanolide \\
\hline 255.232954 & 255.232940 & 0.0539 & 1 & $\mathrm{C}_{16} \mathrm{H}_{32} \mathrm{O}_{2}$ & 25.0550 & 19.8753 & 46.7484 & 28.3596 & Palmitic acid \\
\hline 269.248604 & 269.248597 & 0.0269 & 1 & $\mathrm{C}_{17} \mathrm{H}_{34} \mathrm{O}_{2}$ & 0.9011 & 0.9374 & 1.3521 & 0.5864 & Methyl palmitate \\
\hline 271.227868 & 271.227879 & 0.0396 & 1 & $\mathrm{C}_{16} \mathrm{H}_{32} \mathrm{O}_{3}$ & 0.4615 & 0.6611 & 0.5226 & 0.2946 & Hydroxypalmitic acid \\
\hline
\end{tabular}




\begin{tabular}{|c|c|c|c|c|c|c|c|c|c|}
\hline 281.248604 & 281.248591 & 0.0471 & 2 & $\mathrm{C}_{18} \mathrm{H}_{34} \mathrm{O}_{2}$ & 3.7514 & 4.6453 & 4.5079 & 2.5868 & Oleic acid \\
\hline 283.264254 & 283.264240 & 0.0512 & 1 & $\mathrm{C}_{18} \mathrm{H}_{36} \mathrm{O}_{2}$ & 12.8553 & 2.5077 & 3.8847 & 9.3023 & Steric acid \\
\hline 289.253689 & 289.253636 & 0.1832 & 4 & $\mathrm{C}_{20} \mathrm{H}_{34} \mathrm{O}_{1}$ & 0.0440 & N.D. & 0.0065 & N.D. & Isoabienol \\
\hline 291.232954 & 291.232952 & 0.0069 & 4 & $\mathrm{C}_{19} \mathrm{H}_{32} \mathrm{O}_{2}$ & N.D. & N.D. & 0.0147 & N.D. & 15-Norpinifolic acid \\
\hline 299.201654 & 299.201635 & 0.0652 & 7 & $\mathrm{C}_{20} \mathrm{H}_{28} \mathrm{O}_{2}$ & 1.0363 & 0.8936 & 0.4583 & 0.4346 & Dehydroabietic acid \\
\hline 301.217304 & 301.217305 & 0.0026 & 6 & $\mathrm{C}_{20} \mathrm{H}_{30} \mathrm{O}_{2}$ & 4.3814 & 2.6722 & 14.0433 & 14.8488 & Pimaric acid \\
\hline 301.238433 & 301.238412 & 0.0705 & 1 & $\mathrm{C}_{17} \mathrm{H}_{34} \mathrm{O}_{4}$ & 0.8371 & 1.1883 & 0.6341 & 1.2170 & $\begin{array}{l}\text { 2,3-Dihydroxypropyl } \\
\text { tetradecanoate }\end{array}$ \\
\hline 303.232954 & 303.232931 & 0.0750 & 5 & $\mathrm{C}_{20} \mathrm{H}_{32} \mathrm{O}_{2}$ & 0.2245 & 0.1072 & 0.3229 & 0.1827 & Anticopalic acid \\
\hline 305.248604 & 305.248576 & 0.0925 & 4 & $\mathrm{C}_{20} \mathrm{H}_{34} \mathrm{O}_{2}$ & 0.1440 & 0.2366 & 0.8551 & 0.5622 & 3- $\alpha$-Hydroxymanool \\
\hline 315.196568 & 315.196533 & 0.1102 & 7 & $\mathrm{C}_{20} \mathrm{H}_{28} \mathrm{O}_{3}$ & 0.0637 & 0.1222 & 3.0093 & 0.0101 & Lambertianic acid \\
\hline 317.212218 & 317.212202 & 0.1497 & 6 & $\mathrm{C}_{20} \mathrm{H}_{30} \mathrm{O}_{3}$ & 0.1522 & 0.4172 & 0.0387 & 0.5830 & 12-Hydroxyabietic acid \\
\hline 319.227868 & 319.227845 & 0.0713 & 5 & $\mathrm{C}_{20} \mathrm{H}_{32} \mathrm{O}_{3}$ & 0.2574 & 0.6459 & N.D. & 0.0108 & Isocupressic acid \\
\hline 321.243519 & 321.243501 & 0.0553 & 4 & $\mathrm{C}_{20} \mathrm{H}_{34} \mathrm{O}_{3}$ & 0.0636 & 0.1171 & 0.2147 & 0.0256 & $\begin{array}{l}\text { Imbricatolic acid } \\
\text { (dihydroisocupressic acid) }\end{array}$ \\
\hline 329.269733 & 329.269719 & 0.0440 & 1 & $\mathrm{C}_{19} \mathrm{H}_{38} \mathrm{O}_{4}$ & 1.0562 & 1.5550 & 0.4619 & 0.3422 & $\begin{array}{l}\text { 2,3-Dihydroxypropyl } \\
\text { hexadecanoate }\end{array}$ \\
\hline 331.191483 & 331.191487 & 0.0128 & 7 & $\mathrm{C}_{20} \mathrm{H}_{28} \mathrm{O}_{4}$ & 0.0292 & 0.1459 & 0.0088 & 0.1054 & Pinusolic acid \\
\hline 335.222783 & 335.222770 & 0.0380 & 5 & $\mathrm{C}_{20} \mathrm{H}_{32} \mathrm{O}_{4}$ & 0.8810 & 4.0941 & 0.0551 & 0.3944 & Pinifolic acid \\
\hline 349.202048 & 349.202037 & 0.0315 & 6 & $\mathrm{C}_{20} \mathrm{H}_{30} \mathrm{O}_{5}$ & 0.1645 & 0.2953 & 0.1547 & 0.1180 & Andrographolide \\
\hline 357.301033 & 357.301034 & 0.0028 & 1 & $\mathrm{C}_{21} \mathrm{H}_{42} \mathrm{O}_{4}$ & 1.5215 & 2.6635 & 4.2769 & N.D. & $\begin{array}{l}\text { 2,3-Dihydroxypropyl } \\
\text { octadecanoate }\end{array}$ \\
\hline 361.238433 & 361.238401 & 0.0879 & 6 & $\mathrm{C}_{22} \mathrm{H}_{34} \mathrm{O}_{4}$ & 0.2844 & 0.1568 & 0.9465 & 0.6259 & Acetylisocupressic acid \\
\hline 385.332334 & 385.332326 & 0.0210 & 1 & $\mathrm{C}_{23} \mathrm{H}_{46} \mathrm{O}_{4}$ & 2.0682 & 4.3174 & 1.5368 & 1.2646 & 2,3-dihydroxypropyl eicosanoate \\
\hline 413.363634 & 413.363651 & 0.0417 & 1 & $\mathrm{C}_{25} \mathrm{H}_{50} \mathrm{O}_{4}$ & 2.3874 & 3.9363 & 2.1463 & 2.9763 & 2,3-dihydroxypropyl docosanoate \\
\hline
\end{tabular}

N.D. -Not detected

Table S2. Compounds identified with (-) ESI from conifer needle solvent extracts.

\begin{tabular}{|c|c|c|c|c|c|c|c|}
\hline $\begin{array}{l}\text { Calculated } \\
m / z[\mathrm{M}-\mathrm{H}]^{-}\end{array}$ & $\begin{array}{c}\text { Observed } \\
m / z[\mathrm{M}-\mathrm{H}]^{-}\end{array}$ & $\begin{array}{l}\text { Error } \\
(\mathrm{ppm})\end{array}$ & DBE & $\begin{array}{l}\text { Elemental } \\
\text { formula }\end{array}$ & \multicolumn{2}{|c|}{ Relative intensity (\%) } & \multirow[t]{2}{*}{ Putative compound(s) } \\
\hline & & & & & Hexane & Toluene & \\
\hline 121.029503 & 121.029503 & 0.0000 & 5 & $\mathrm{C}_{7} \mathrm{H}_{6} \mathrm{O}_{2}$ & 0.0090 & 0.0275 & Benzoic acid \\
\hline 137.024418 & 137.024422 & 0.0292 & 5 & $\mathrm{C}_{7} \mathrm{H}_{6} \mathrm{O}_{3}$ & 0.0056 & 0.0933 & Salicyclic acid \\
\hline 143.107753 & 143.107757 & 0.0280 & 1 & $\mathrm{C}_{8} \mathrm{H}_{16} \mathrm{O}_{2}$ & 0.0140 & 0.0190 & Octanoic acid \\
\hline 145.087018 & 145.087027 & 0.0586 & 1 & $\mathrm{C}_{7} \mathrm{H}_{14} \mathrm{O}_{3}$ & 0.0107 & 0.0492 & 2-Hydroxyheptanoic acid \\
\hline 149.045547 & 149.045556 & 0.0604 & 1 & $\mathrm{C}_{5} \mathrm{H}_{10} \mathrm{O}_{5}$ & 0.0573 & N.D. & Arabinose \\
\hline 151.040068 & 151.040074 & 0.0397 & 5 & $\mathrm{C}_{8} \mathrm{H}_{8} \mathrm{O}_{3}$ & 0.0066 & 0.0229 & 4-hydroxyphenyl acetic acid \\
\hline 163.061197 & 163.060648 & 0.0552 & 1 & $\mathrm{C}_{6} \mathrm{H}_{12} \mathrm{O}_{5}$ & 0.0126 & N.D. & Rhamnose \\
\hline 167.107753 & 167.107770 & 0.1017 & 3 & $\mathrm{C}_{10} \mathrm{H}_{16} \mathrm{O}_{2}$ & 0.0107 & 0.2334 & Geranic acid \\
\hline 171.139053 & 171.139068 & 0.0819 & 1 & $\mathrm{C}_{10} \mathrm{H}_{20} \mathrm{O}_{2}$ & 0.0056 & N.D. & Decanoic acid \\
\hline 179.056112 & 179.056124 & 0.0540 & 1 & $\mathrm{C}_{6} \mathrm{H}_{12} \mathrm{O}_{6}$ & 0.2072 & 0.0153 & Frutose \\
\hline 183.102668 & 183.102689 & 0.0942 & 3 & $\mathrm{C}_{10} \mathrm{H}_{16} \mathrm{O}_{3}$ & N.D. & 0.0219 & Pinonic acid \\
\hline 183.139053 & 183.139068 & 0.0518 & 2 & $\mathrm{C}_{11} \mathrm{H}_{20} \mathrm{O}_{2}$ & 0.0513 & N.D. & Z-3-Hexenyl isovalerate \\
\hline 185.081932 & 185.081942 & 0.0906 & 3 & $\mathrm{C}_{9} \mathrm{H}_{14} \mathrm{O}_{4}$ & 0.0075 & 0.0286 & Pinic acid \\
\hline 191.056112 & 191.056094 & 0.0276 & 2 & $\mathrm{C}_{7} \mathrm{H}_{12} \mathrm{O}_{6}$ & 3.4664 & 0.3052 & Quinic acid \\
\hline 193.050632 & 193.050642 & 0.0536 & 6 & $\mathrm{C}_{10} \mathrm{H}_{10} \mathrm{O}_{4}$ & N.D. & 0.0473 & Ferulic acid \\
\hline 193.071762 & 193.071780 & 0.0047 & 1 & $\mathrm{C}_{7} \mathrm{H}_{14} \mathrm{O}_{6}$ & 0.0523 & 0.0637 & Pinitol \\
\hline
\end{tabular}




\begin{tabular}{|c|c|c|c|c|c|c|c|}
\hline 199.170354 & 199.170360 & 0.0813 & 1 & $\mathrm{C}_{12} \mathrm{H}_{24} \mathrm{O}_{2}$ & 0.0281 & 0.0309 & Lauric acid \\
\hline 205.159789 & 205.159778 & 0.0023 & 4 & $\mathrm{C}_{14} \mathrm{H}_{22} \mathrm{O}_{1}$ & 0.0169 & N.D. & Alpha irone \\
\hline 213.186004 & 213.186005 & 0.1155 & 1 & $\mathrm{C}_{13} \mathrm{H}_{26} \mathrm{O}_{2}$ & 0.0063 & N.D. & Tridecanoic acid \\
\hline 215.165268 & 215.165286 & 0.1285 & 1 & $\mathrm{C}_{12} \mathrm{H}_{24} \mathrm{O}_{3}$ & 0.0185 & 0.0297 & Sabinic acid (hydroxylauric acid) \\
\hline 221.081932 & 221.081933 & 0.1501 & 6 & $\mathrm{C}_{12} \mathrm{H}_{14} \mathrm{O}_{4}$ & 0.0052 & 0.0159 & Dillapiole \\
\hline 225.092103 & 225.092129 & 0.1155 & 9 & $\mathrm{C}_{15} \mathrm{H}_{14} \mathrm{O}_{2}$ & 0.0337 & N.D. & Flavon-3-ol \\
\hline 227.201654 & 227.201683 & 0.1276 & 1 & $\mathrm{C}_{14} \mathrm{H}_{28} \mathrm{O}_{2}$ & N.D. & 0.0280 & Myristic acid \\
\hline 239.201654 & 239.201709 & 0.2299 & 2 & $\mathrm{C}_{15} \mathrm{H}_{28} \mathrm{O}_{2}$ & 0.0059 & N.D. & Methyl isovalerate \\
\hline 241.217304 & 241.217335 & 0.1285 & 1 & $\mathrm{C}_{15} \mathrm{H}_{30} \mathrm{O}_{2}$ & 0.0121 & N.D. & Pentadecanoic acid \\
\hline 253.217304 & 253.217335 & 0.1501 & 2 & $\mathrm{C}_{16} \mathrm{H}_{30} \mathrm{O}_{2}$ & 0.0165 & 0.0165 & Palmitoleic acid \\
\hline 255.232954 & 255.232951 & 0.0137 & 1 & $\mathrm{C}_{16} \mathrm{H}_{32} \mathrm{O}_{2}$ & 0.2940 & 0.1350 & Palmitic acid \\
\hline 269.248604 & 269.248559 & 0.1671 & 1 & $\mathrm{C}_{17} \mathrm{H}_{34} \mathrm{O}_{2}$ & 0.0153 & N.D. & Methyl palmitate \\
\hline 271.227868 & 271.227872 & 0.0129 & 1 & $\mathrm{C}_{16} \mathrm{H}_{32} \mathrm{O}_{3}$ & 0.1633 & 0.2314 & Hydroxypalmitic acid \\
\hline 277.217304 & 277.217325 & 0.0739 & 4 & $\mathrm{C}_{18} \mathrm{H}_{30} \mathrm{O}_{2}$ & 0.3064 & 0.0501 & Linolenic acid \\
\hline 279.232954 & 279.232967 & 0.0448 & 3 & $\mathrm{C}_{18} \mathrm{H}_{32} \mathrm{O}_{2}$ & 0.0727 & 0.0281 & Linoleic acid \\
\hline 281.248604 & 281.248559 & 0.0181 & 2 & $\mathrm{C}_{18} \mathrm{H}_{34} \mathrm{O}_{2}$ & 0.0357 & N.D. & Oleic acid \\
\hline 283.264254 & 283.264232 & 0.0777 & 1 & $\mathrm{C}_{18} \mathrm{H}_{36} \mathrm{O}_{2}$ & 0.2192 & 0.0757 & Stearic acid \\
\hline 285.040462 & 285.040469 & 0.0246 & 11 & $\mathrm{C}_{15} \mathrm{H}_{10} \mathrm{O}_{6}$ & N.D. & 0.0334 & Kaempferol \\
\hline 291.232954 & 291.232955 & 0.0034 & 4 & $\mathrm{C}_{19} \mathrm{H}_{32} \mathrm{O}_{2}$ & 0.0092 & N.D. & 15-Norpinifolic acid \\
\hline 297.279904 & 297.279948 & 0.1480 & 1 & $\mathrm{C}_{19} \mathrm{H}_{38} \mathrm{O}_{2}$ & 0.0064 & N.D. & Nonadecanoic acid \\
\hline 299.165268 & 299.165246 & 0.0735 & 8 & $\mathrm{C}_{19} \mathrm{H}_{24} \mathrm{O}_{3}$ & 0.0174 & 0.0191 & Z-Bornyl-p-coumarate \\
\hline 299.201654 & 299.201619 & 0.1170 & 7 & $\mathrm{C}_{20} \mathrm{H}_{28} \mathrm{O}_{2}$ & 0.8487 & 0.6294 & Dehydroabietic acid \\
\hline 301.035376 & 301.035324 & 0.1727 & 11 & $\mathrm{C}_{15} \mathrm{H}_{10} \mathrm{O}_{7}$ & N.D. & 0.0175 & Quercetin \\
\hline 301.217304 & 301.217288 & 0.0548 & 6 & $\mathrm{C}_{20} \mathrm{H}_{30} \mathrm{O}_{2}$ & 6.7897 & 3.5646 & Abietic acid \\
\hline 301.238433 & 301.238377 & 0.1859 & 1 & $\mathrm{C}_{17} \mathrm{H}_{34} \mathrm{O}_{4}$ & 0.0877 & N.D. & 2,3-Dihydroxypropyl tetradecanoate \\
\hline 303.051026 & 303.051083 & 0.1881 & 10 & $\mathrm{C}_{15} \mathrm{H}_{12} \mathrm{O}_{7}$ & N.D. & 0.0209 & Taxifolin \\
\hline 303.232954 & 303.232900 & 0.1797 & 5 & $\mathrm{C}_{20} \mathrm{H}_{32} \mathrm{O}_{2}$ & 0.0347 & 0.0191 & Anticopalic acid \\
\hline 305.248604 & 305.248533 & 0.2326 & 4 & $\mathrm{C}_{20} \mathrm{H}_{34} \mathrm{O}_{2}$ & 0.0273 & N.D. & $3-\alpha$-Hydroxy manool \\
\hline 311.295554 & 311.295523 & 0.0996 & 1 & $\mathrm{C}_{20} \mathrm{H}_{40} \mathrm{O}_{2}$ & 0.0661 & 0.0258 & Arachidic acid \\
\hline 313.180918 & 313.180901 & 0.0559 & 8 & $\mathrm{C}_{20} \mathrm{H}_{26} \mathrm{O}_{3}$ & 0.0071 & 0.0251 & 7-Oxodehydroabietic acid \\
\hline 315.051026 & 315.051018 & 0.0254 & 11 & $\mathrm{C}_{16} \mathrm{H}_{12} \mathrm{O}_{7}$ & N.D. & 0.0502 & Isorhamnetin \\
\hline 315.196568 & 315.196534 & 0.1079 & 7 & $\mathrm{C}_{20} \mathrm{H}_{28} \mathrm{O}_{3}$ & 0.0886 & 0.1914 & Lambertianic acid \\
\hline 317.212218 & 317.212208 & 0.0315 & 6 & $\mathrm{C}_{20} \mathrm{H}_{30} \mathrm{O}_{3}$ & 0.4904 & 1.1300 & 12-Hydroxyabietic acid \\
\hline 319.227868 & 319.227841 & 0.0846 & 5 & $\mathrm{C}_{20} \mathrm{H}_{32} \mathrm{O}_{3}$ & 1.3126 & 2.8905 & Isocupressic acid \\
\hline 321.243519 & 321.243482 & 0.1167 & 4 & $\mathrm{C}_{20} \mathrm{H}_{34} \mathrm{O}_{3}$ & 0.1606 & 0.4492 & Imbricatolic acid (dihydroisocupressic acid) \\
\hline 329.269733 & 329.269684 & 0.1488 & 1 & $\mathrm{C}_{19} \mathrm{H}_{38} \mathrm{O}_{4}$ & 0.0118 & 0.2700 & 2,3-Dihydroxypropyl hexadecanoate \\
\hline 331.191483 & 331.191442 & 0.1238 & 7 & $\mathrm{C}_{20} \mathrm{H}_{28} \mathrm{O}_{4}$ & 0.0465 & N.D. & Pinusolic acid \\
\hline 333.207133 & 333.207094 & 0.1185 & 6 & $\mathrm{C}_{20} \mathrm{H}_{30} \mathrm{O}_{4}$ & 9.5100 & 10.8080 & Dehydropinifolic acid \\
\hline 335.222783 & 335.222739 & 0.1313 & 5 & $\mathrm{C}_{20} \mathrm{H}_{32} \mathrm{O}_{4}$ & 8.4787 & 27.8598 & Pinifolic acid \\
\hline 339.326854 & 339.326797 & 0.1695 & 1 & $\mathrm{C}_{22} \mathrm{H}_{44} \mathrm{O}_{2}$ & 0.0516 & 0.0178 & Behenic acid \\
\hline 341.108935 & 341.108914 & 0.0616 & 2 & $\mathrm{C}_{12} \mathrm{H}_{22} \mathrm{O}_{11}$ & 0.1491 & 0.0586 & Sucrose \\
\hline 345.207133 & 345.207115 & 0.0521 & 7 & $\mathrm{C}_{21} \mathrm{H}_{30} \mathrm{O}_{4}$ & 0.0358 & 0.0442 & Pinusolide \\
\hline 349.202048 & 349.202019 & 0.0845 & 6 & $\mathrm{C}_{20} \mathrm{H}_{30} \mathrm{O}_{5}$ & 0.1486 & 0.4292 & Andrographolide \\
\hline
\end{tabular}




\begin{tabular}{|c|c|c|c|c|c|c|c|}
\hline 349.238433 & 349.238396 & 0.1059 & 5 & $\mathrm{C}_{21} \mathrm{H}_{34} \mathrm{O}_{4}$ & 33.1969 & 44.5993 & Methyl pinifolate \\
\hline 353.342504 & 353.342436 & 0.1924 & 1 & $\mathrm{C}_{23} \mathrm{H}_{46} \mathrm{O}_{2}$ & 0.0067 & N.D. & Tricocosanic acid \\
\hline 355.321769 & 355.321733 & 0.1027 & 1 & $\mathrm{C}_{22} \mathrm{H}_{44} \mathrm{O}_{3}$ & 0.0238 & 0.0494 & Hydroxydocosanoic acid \\
\hline 357.134362 & 357.134358 & 0.0112 & 10 & $\mathrm{C}_{20} \mathrm{H}_{22} \mathrm{O}_{6}$ & N.D. & 0.0440 & Matairesinol \\
\hline 357.301033 & 357.300986 & 0.1315 & 1 & $\mathrm{C}_{21} \mathrm{H}_{42} \mathrm{O}_{4}$ & 0.0049 & 0.0193 & 2,3-Dihydroxypropyl octadecanoate \\
\hline 361.238433 & 361.238406 & 0.0747 & 6 & $\mathrm{C}_{22} \mathrm{H}_{34} \mathrm{O}_{4}$ & 2.3195 & 1.6923 & Acetylisocupressic acid \\
\hline 363.254083 & 363.254057 & 0.0730 & 5 & $\mathrm{C}_{22} \mathrm{H}_{36} \mathrm{O}_{4}$ & 0.7503 & 1.1065 & Dimethyl pinifolate \\
\hline 365.196962 & 365.196957 & 0.0137 & 6 & $\mathrm{C}_{20} \mathrm{H}_{30} \mathrm{O}_{6}$ & 1.0325 & N.D. & $\begin{array}{l}\text { 7-Oxo-12 } \alpha, 13 \beta, 15 \text {-trihydroxyabiet-8(14)-en- } \\
\text { 18-oic acid }\end{array}$ \\
\hline 371.11950 & 371.119478 & 0.0593 & 2 & $\mathrm{C}_{13} \mathrm{H}_{24} \mathrm{O}_{12}$ & 4.9789 & N.D. & 6-O-Heptopyranosyl-d-glucopyranose \\
\hline 373.129277 & 373.129310 & 0.0643 & 10 & $\mathrm{C}_{20} \mathrm{H}_{22} \mathrm{O}_{7}$ & N.D. & 0.0244 & Hydroxymatairesinol \\
\hline 381.373804 & 381.373829 & 0.0656 & 1 & $\mathrm{C}_{25} \mathrm{H}_{50} \mathrm{O}_{2}$ & 0.0063 & N.D. & Pentacosanoic acid \\
\hline 385.332334 & 385.332301 & 0.0830 & 1 & $\mathrm{C}_{23} \mathrm{H}_{46} \mathrm{O}_{4}$ & 0.0719 & 0.2834 & 2,3-Dihydroxypropyl eicosanoate \\
\hline 395.389454 & 395.389407 & 0.1189 & 1 & $\mathrm{C}_{26} \mathrm{H}_{52} \mathrm{O}_{2}$ & 0.0175 & N.D. & Hexacosanic acid \\
\hline 409.259562 & 409.259559 & 0.0073 & 5 & $\mathrm{C}_{23} \mathrm{H}_{38} \mathrm{O}_{6}$ & 23.8423 & 1.6595 & Vitetrifolin D \\
\hline 413.363634 & 413.363648 & 0.0339 & 1 & $\mathrm{C}_{25} \mathrm{H}_{50} \mathrm{O}_{4}$ & 0.0327 & 0.1216 & 2,3-Dihydroxypropyl docosanoate \\
\hline 419.243912 & 419.243883 & 0.0704 & 7 & $\mathrm{C}_{24} \mathrm{H}_{36} \mathrm{O}_{6}$ & 0.0841 & 0.2778 & Succinylisocupressic acid \\
\hline 423.420755 & 423.420773 & 0.0425 & 1 & $\mathrm{C}_{28} \mathrm{H}_{56} \mathrm{O}_{2}$ & 0.0287 & N.D. & Octacosanic acid \\
\hline 451.452055 & 451.452026 & 0.0642 & 1 & $\mathrm{C}_{30} \mathrm{H}_{60} \mathrm{O}_{2}$ & 0.0320 & N.D. & Triacontanoic acid \\
\hline 463.08820 & 463.088170 & 0.0648 & 12 & $\mathrm{C}_{21} \mathrm{H}_{20} \mathrm{O}_{12}$ & 0.0071 & N.D. & Isoquercetin \\
\hline 479.483355 & 479.483310 & 0.0939 & 1 & $\mathrm{C}_{32} \mathrm{H}_{64} \mathrm{O}_{2}$ & 0.0103 & N.D. & Dotriacontanoic acid \\
\hline 503.161758 & 503.161703 & 0.1093 & 3 & $\mathrm{C}_{18} \mathrm{H}_{32} \mathrm{O}_{16}$ & 0.0087 & N.D. & Raffinose \\
\hline
\end{tabular}

N.D. -Not detected

Table S3. Compounds identified with $(+)$ APPI from conifer needle essential oils

\begin{tabular}{|c|c|c|c|c|c|c|c|c|c|}
\hline $\begin{array}{l}\text { Calculated } \\
m / z[\mathrm{M}+\mathrm{H}]^{+}\end{array}$ & $\begin{array}{c}\text { Observed } \\
m / z[\mathrm{M}+\mathrm{H}]^{+}\end{array}$ & $\begin{array}{l}\text { Error } \\
(\mathrm{ppm})\end{array}$ & DBE & $\begin{array}{l}\text { Elemental } \\
\text { formula }\end{array}$ & \multicolumn{4}{|c|}{ Relative intensity (\%) } & Putative compound(s) \\
\hline & & & & & Pine & Spruce & Larch & Juniper & \\
\hline 121.101177 & 121.101168 & 0.0716 & 4 & $\mathrm{C}_{9} \mathrm{H}_{12}$ & 0.9168 & 1.0286 & 0.4138 & 1.0538 & 1,2,4-Trimethylbenzene \\
\hline 133.101177 & 133.101175 & 0.0175 & 5 & $\mathrm{C}_{10} \mathrm{H}_{12}$ & 0.1279 & 0.1079 & 0.2609 & 0.1249 & 3,5-Dimethylstyrene \\
\hline 135.080441 & 135.080446 & 0.0370 & 5 & $\mathrm{C}_{9} \mathrm{H}_{10} \mathrm{O}_{1}$ & 0.0348 & 0.0289 & 0.1455 & 0.0129 & Chavicol \\
\hline 135.116827 & 135.116826 & 0.0074 & 4 & $\mathrm{C}_{10} \mathrm{H}_{14}$ & 0.4837 & 2.0141 & 0.4364 & 0.9049 & Verbenene \\
\hline 137.132477 & 137.132464 & 0.0972 & 3 & $\mathrm{C}_{10} \mathrm{H}_{16}$ & 5.9086 & 21.0899 & 0.5062 & 11.0749 & Pinene \\
\hline 139.111742 & 139.111740 & 0.0144 & 3 & $\mathrm{C}_{9} \mathrm{H}_{14} \mathrm{O}_{1}$ & 0.0640 & 3.5497 & 0.1218 & 0.0714 & Sebina Ketone \\
\hline 149.096091 & 149.096079 & 0.0827 & 5 & $\mathrm{C}_{10} \mathrm{H}_{12} \mathrm{O}_{1}$ & 0.0973 & 0.0693 & 0.1944 & 0.0694 & Methyl chavicol \\
\hline 151.111742 & 151.111719 & 0.1522 & 4 & $\mathrm{C}_{10} \mathrm{H}_{14} \mathrm{O}_{1}$ & 1.6385 & 0.8118 & 1.1858 & 0.3063 & Verbenone \\
\hline 153.127392 & 153.127376 & 0.1023 & 3 & $\mathrm{C}_{10} \mathrm{H}_{16} \mathrm{O}_{1}$ & 0.2257 & 4.7042 & 0.2309 & 0.4604 & $\begin{array}{l}\text { Methyl thymol, Thujone, } \\
\text { Piperitone }\end{array}$ \\
\hline 155.143042 & 155.143032 & 0.0677 & 2 & $\mathrm{C}_{10} \mathrm{H}_{18} \mathrm{O}_{1}$ & N.D. & 0.1961 & N.D. & 0.0114 & $\begin{array}{l}\text { Borneol, Citronellal, } \\
\text { Eucalyptol, linalool, Caraneol }\end{array}$ \\
\hline 161.132477 & 161.132444 & 0.2027 & 5 & $\mathrm{C}_{12} \mathrm{H}_{16}$ & 2.2558 & 1.1657 & 9.6802 & 6.1491 & 3-Methyl-2-phenyl-2-pentene \\
\hline 163.075356 & 163.075350 & 0.0399 & 6 & $\mathrm{C}_{10} \mathrm{H}_{10} \mathrm{O}_{2}$ & N.D. & 0.0605 & 0.0460 & 0.0107 & Safrole \\
\hline 165.091006 & 165.091005 & 0.0081 & 5 & $\mathrm{C}_{10} \mathrm{H}_{12} \mathrm{O}_{2}$ & 0.0955 & 0.0486 & 0.2249 & 0.0408 & 2-Phenylethyl acetate \\
\hline 165.127392 & 165.127384 & 0.0515 & 4 & $\mathrm{C}_{11} \mathrm{H}_{16} \mathrm{O}_{1}$ & 0.0620 & 0.0647 & 0.0956 & 0.0679 & Thymol methyl ether \\
\hline 167.106656 & 167.106653 & 0.0160 & 4 & $\mathrm{C}_{10} \mathrm{H}_{14} \mathrm{O}_{2}$ & 0.2318 & 0.1488 & 0.6396 & 0.0608 & Perillic acid \\
\hline 169.122306 & 169.122300 & 0.0335 & 3 & $\mathrm{C}_{10} \mathrm{H}_{16} \mathrm{O}_{2}$ & 0.0606 & 0.2716 & 0.3167 & 0.0847 & Geranic acid \\
\hline 171.137956 & 171.137952 & 0.0263 & 2 & $\mathrm{C}_{10} \mathrm{H}_{18} \mathrm{O}_{2}$ & N.D. & 0.0453 & 0.0090 & 0.0374 & Citronellic acid \\
\hline
\end{tabular}




\begin{tabular}{|c|c|c|c|c|c|c|c|c|c|}
\hline 179.106656 & 179.106657 & 0.0037 & 5 & $\mathrm{C}_{11} \mathrm{H}_{14} \mathrm{O}_{2}$ & 0.0159 & 0.0351 & 0.0825 & 0.0208 & Methyl eugenol \\
\hline 181.085921 & 181.085915 & 0.0331 & 5 & $\mathrm{C}_{10} \mathrm{H}_{12} \mathrm{O}_{3}$ & N.D. & 0.0096 & 0.0670 & N.D. & Coniferyl alcohol \\
\hline 183.137956 & 183.137943 & 0.0710 & 3 & $\mathrm{C}_{11} \mathrm{H}_{18} \mathrm{O}_{2}$ & 0.0345 & 0.0890 & 0.0523 & 0.0565 & Geranyl formate \\
\hline 193.085921 & 193.085900 & 0.1088 & 6 & $\mathrm{C}_{11} \mathrm{H}_{12} \mathrm{O}_{3}$ & N.D. & N.D. & 0.0129 & N.D. & Myristicin \\
\hline 193.122306 & 193.122302 & 0.0207 & 5 & $\mathrm{C}_{12} \mathrm{H}_{16} \mathrm{O}_{2}$ & 0.0670 & 0.1862 & 0.3605 & 0.0532 & $\beta$-Phenylethyl isobutyrate \\
\hline 197.153606 & 197.153568 & 0.1927 & 3 & $\mathrm{C}_{12} \mathrm{H}_{20} \mathrm{O}_{2}$ & N.D. & 0.0062 & 0.0080 & N.D. & Geranyl acetate \\
\hline 201.163777 & 201.163745 & 0.1591 & 6 & $\mathrm{C}_{15} \mathrm{H}_{20}$ & 0.6235 & 0.2380 & 0.7323 & 0.1514 & Calorene \\
\hline 203.179427 & 203.179378 & 0.2428 & 5 & $\mathrm{C}_{15} \mathrm{H}_{22}$ & 2.7711 & 4.2812 & 2.1884 & 1.8958 & Calamenene \\
\hline 205.158692 & 205.158671 & 0.1024 & 5 & $\mathrm{C}_{14} \mathrm{H}_{20} \mathrm{O}_{1}$ & 2.7894 & 0.8269 & 1.9393 & 0.0915 & Khusimone \\
\hline 205.195077 & 205.195005 & 0.3509 & 4 & $\mathrm{C}_{15} \mathrm{H}_{24}$ & 56.4577 & 32.5175 & 56.6424 & 58.6900 & Germacrene D, Caryophyllene \\
\hline 207.137956 & 207.137956 & 0.0016 & 5 & $\mathrm{C}_{13} \mathrm{H}_{18} \mathrm{O}_{2}$ & 0.1590 & 0.1029 & 0.2404 & 0.0416 & 3-Methyl-2-phenylethyl ester \\
\hline 207.174342 & 207.174319 & 0.1110 & 4 & $\mathrm{C}_{14} \mathrm{H}_{22} \mathrm{O}$ & 2.4766 & 0.4495 & 3.9191 & 2.2276 & Alpha irone \\
\hline 209.117221 & 209.117204 & 0.0829 & 5 & $\mathrm{C}_{12} \mathrm{H}_{16} \mathrm{O}_{3}$ & N.D. & 0.0538 & 0.1549 & 0.0299 & Pinchotene acetate \\
\hline 211.169256 & 211.169248 & 0.0395 & 3 & $\mathrm{C}_{13} \mathrm{H}_{22} \mathrm{O}_{2}$ & 0.0245 & 0.0905 & 0.0486 & 0.0191 & Isobornyl propaniote \\
\hline 217.158692 & 217.158686 & 0.0299 & 6 & $\mathrm{C}_{15} \mathrm{H}_{20} \mathrm{O}_{1}$ & 1.3740 & 0.5028 & 1.6941 & 0.2251 & Hexyl cinnamaldehyde \\
\hline 217.195077 & 217.195069 & 0.0353 & 5 & $\mathrm{C}_{16} \mathrm{H}_{24}$ & 1.5713 & 3.1080 & 0.1362 & 5.4005 & Vulgraon B \\
\hline 219.174342 & 219.174303 & 0.1779 & 5 & $\mathrm{C}_{15} \mathrm{H}_{22} \mathrm{O}_{1}$ & 7.0388 & 2.8140 & 8.1671 & 1.1068 & Nootkatone \\
\hline 221.153606 & 221.153593 & 0.0573 & 5 & $\mathrm{C}_{14} \mathrm{H}_{20} \mathrm{O}_{2}$ & 1.1009 & 0.1587 & 1.5341 & 0.0915 & Phenylethyl hexanoate \\
\hline 221.189992 & 221.189962 & 0.1341 & 4 & $\mathrm{C}_{15} \mathrm{H}_{24} \mathrm{O}_{1}$ & 3.0924 & 5.9276 & 2.5463 & 2.1145 & $\begin{array}{l}\text { Caryophyllene oxide, Farnasal, } \\
\text { Spathulenol }\end{array}$ \\
\hline 223.075356 & 223.075371 & 0.0672 & 11 & $\mathrm{C}_{15} \mathrm{H}_{10} \mathrm{O}_{2}$ & N.D. & 0.0062 & N.D. & N.D. & Aurone, Flavone \\
\hline 223.096485 & 223.096450 & 0.1569 & 6 & $\mathrm{C}_{12} \mathrm{H}_{14} \mathrm{O}_{4}$ & N.D. & 0.0082 & 0.0254 & N.D. & Apiole \\
\hline 223.205642 & 223.205644 & 0.0105 & 3 & $\mathrm{C}_{15} \mathrm{H}_{26} \mathrm{O}_{1}$ & 0.0408 & 0.0958 & 0.2454 & 0.0931 & Germacrene D-4-ol \\
\hline 225.184906 & 225.184889 & 0.0755 & 3 & $\mathrm{C}_{14} \mathrm{H}_{24} \mathrm{O}_{2}$ & 0.0206 & 0.0467 & 0.0517 & 0.0226 & Bornyl isobutyrate \\
\hline 237.184906 & 237.184902 & 0.0169 & 4 & $\mathrm{C}_{15} \mathrm{H}_{24} \mathrm{O}_{2}$ & 1.6275 & 0.6122 & 1.7964 & 0.4036 & Myrtenyl isovalerate \\
\hline 239.164171 & 239.164157 & 0.0599 & 4 & $\mathrm{C}_{14} \mathrm{H}_{22} \mathrm{O}_{3}$ & 0.0939 & 0.0273 & 0.3354 & 0.0297 & Pinchotene acetate \\
\hline 239.200557 & 239.200565 & 0.0334 & 3 & $\mathrm{C}_{15} \mathrm{H}_{26} \mathrm{O}_{2}$ & 0.0261 & 0.0883 & 0.0389 & 0.0397 & Bornyl-2-methylbutyrate \\
\hline 255.210727 & 255.210745 & 0.0705 & 7 & $\mathrm{C}_{19} \mathrm{H}_{26}$ & N.D. & 0.2620 & 0.1356 & 0.2895 & $\begin{array}{l}\text { Norabieta-4(18),8,11,13- } \\
\text { tetraene }\end{array}$ \\
\hline 257.226377 & 257.226391 & 0.0557 & 6 & $\mathrm{C}_{19} \mathrm{H}_{28}$ & N.D. & 1.3167 & 0.1103 & 0.3278 & Norabietatriene \\
\hline 271.242027 & 271.241958 & 0.2532 & 6 & $\mathrm{C}_{20} \mathrm{H}_{30}$ & 0.9916 & 2.1543 & 0.2152 & 2.0669 & Abieta-8,11,13-triene \\
\hline 273.257677 & 273.257649 & 0.1025 & 5 & $\mathrm{C}_{20} \mathrm{H}_{32}$ & 2.8188 & 4.9122 & 0.1459 & 1.3402 & Abietadiene \\
\hline 285.221292 & 285.221284 & 0.0269 & 7 & $\mathrm{C}_{20} \mathrm{H}_{28} \mathrm{O}_{1}$ & 0.3728 & 0.3426 & 0.2719 & 0.2837 & Dehydroabietal \\
\hline 287.236942 & 287.236908 & 0.1184 & 6 & $\mathrm{C}_{20} \mathrm{H}_{30} \mathrm{O}_{1}$ & 0.7638 & 1.2838 & 0.3542 & 0.9583 & Ferruginol \\
\hline 289.252592 & 289.252589 & 0.0104 & 5 & $\mathrm{C}_{20} \mathrm{H}_{32} \mathrm{O}_{1}$ & 0.2117 & 0.6814 & 0.0950 & 0.3281 & Isopimarol \\
\hline 291.268242 & 291.268250 & 0.0257 & 4 & $\mathrm{C}_{20} \mathrm{H}_{34} \mathrm{O}_{1}$ & N.D. & 0.0314 & 0.0132 & 0.0310 & Isoabienal \\
\hline 293.247507 & 293.247523 & 0.0529 & 4 & $\mathrm{C}_{19} \mathrm{H}_{32} \mathrm{O}_{2}$ & N.D. & 0.0111 & 0.0088 & 0.0084 & 15-Norpinifolic acid \\
\hline 301.179821 & 301.179831 & 0.0321 & 8 & $\mathrm{C}_{19} \mathrm{H}_{24} \mathrm{O}_{3}$ & 0.0305 & 0.0261 & 0.0085 & 0.0653 & Z-Bornyl-p-coumarate \\
\hline 303.231857 & 303.231830 & 0.0890 & 6 & $\mathrm{C}_{20} \mathrm{H}_{30} \mathrm{O}_{2}$ & 0.4327 & 0.3394 & 0.3379 & 0.2148 & Abietic acid \\
\hline 305.247507 & 305.247468 & 0.1278 & 5 & $\mathrm{C}_{20} \mathrm{H}_{32} \mathrm{O}_{2}$ & 0.0579 & 0.3791 & 0.2433 & 0.1525 & Anticopalic acid \\
\hline 315.231857 & 315.231865 & 0.0254 & 7 & $\mathrm{C}_{21} \mathrm{H}_{30} \mathrm{O}_{2}$ & 0.0935 & 0.1261 & 0.1918 & 0.0381 & Methyl dehydroabietate \\
\hline 317.211121 & 317.211118 & 0.0095 & 7 & $\mathrm{C}_{20} \mathrm{H}_{28} \mathrm{O}_{3}$ & 0.1307 & 0.0626 & 0.0367 & 0.1696 & Lambertianic acid \\
\hline 319.226771 & 319.226527 & 0.0052 & 6 & $\mathrm{C}_{20} \mathrm{H}_{30} \mathrm{O}_{3}$ & 0.1944 & 0.0907 & 0.0353 & 0.1792 & 3-Oxoanticopalic acid \\
\hline 317.247507 & 319.263769 & 0.0620 & 6 & $\mathrm{C}_{21} \mathrm{H}_{32} \mathrm{O}_{2}$ & 0.0326 & 0.0618 & 0.0761 & 0.0298 & Methyl sandaracopimarate \\
\hline 319.263157 & 319.263165 & 0.0261 & 5 & $\mathrm{C}_{21} \mathrm{H}_{34} \mathrm{O}_{2}$ & 0.0326 & 0.0334 & 0.0227 & 0.0427 & Methyl arachidonate \\
\hline 321.242421 & 321.242394 & 0.0830 & 5 & $\mathrm{C}_{20} \mathrm{H}_{32} \mathrm{O}_{3}$ & 0.0876 & 0.0685 & 0.0238 & N.D. & Isocupressic acid \\
\hline 323.258071 & 323.258082 & 0.0340 & 4 & $\mathrm{C}_{20} \mathrm{H}_{34} \mathrm{O}_{3}$ & N.D. & 0.0247 & N.D. & 0.0249 & $\begin{array}{l}\text { Imbricatolic acid } \\
\text { (dihydroisocupressic acid) }\end{array}$ \\
\hline 331.226771 & 331.226784 & 0.0392 & 7 & $\mathrm{C}_{21} \mathrm{H}_{30} \mathrm{O}_{3}$ & 0.0431 & 0.0315 & 0.0914 & 0.0099 & Methyl daniellate \\
\hline 333.206036 & 333.206998 & 0.1150 & 7 & $\mathrm{C}_{20} \mathrm{H}_{28} \mathrm{O}_{4}$ & 0.0542 & 0.0194 & 0.0088 & 0.0733 & Pinosolide \\
\hline
\end{tabular}




\begin{tabular}{|l|l|l|l|l|l|l|l|l|l|}
\hline 337.237336 & 337.237326 & 0.0297 & 5 & $\mathrm{C}_{20} \mathrm{H}_{32} \mathrm{O}_{4}$ & 0.0222 & 0.0279 & 0.0241 & N.D & Pinifolic acid \\
\hline 351.216601 & 351.216617 & 0.0456 & 6 & $\mathrm{C}_{20} \mathrm{H}_{30} \mathrm{O}_{5}$ & 0.0207 & 0.0076 & 0.0224 & N.D & Andrographolide \\
\hline 363.252986 & 363.252934 & 0.1432 & 6 & $\mathrm{C}_{22} \mathrm{H}_{34} \mathrm{O}_{4}$ & N.D. & 0.0177 & N.D. & 0.0194 & Acetylisocupressic acid \\
\hline
\end{tabular}

N.D. - Not detected

Table S4. Compounds identified with $(+)$ APPI from conifer needle solvent extracts

\begin{tabular}{|c|c|c|c|c|c|c|c|}
\hline $\begin{array}{l}\text { Calculated } \\
m / z[\mathrm{M}]^{+\bullet}\end{array}$ & $\begin{array}{l}\text { Observed } \\
m / z[\mathrm{M}]^{+\bullet}\end{array}$ & $\begin{array}{l}\text { Error } \\
(\mathrm{ppm})\end{array}$ & DBE & $\begin{array}{c}\text { Elemental } \\
\text { formula }\end{array}$ & \multicolumn{2}{|c|}{ Relative intensity (\%) } & \multirow[t]{2}{*}{ Putative compound(s) } \\
\hline & & & & & Hexane & Toluene & \\
\hline 134.072616 & 134.072608 & 0.0597 & 5 & $\mathrm{C}_{9} \mathrm{H}_{10} \mathrm{O}_{1}$ & 0.0219 & N.D. & Chavicol \\
\hline 134.109002 & 134.109005 & 0.0186 & 4 & $\mathrm{C}_{10} \mathrm{H}_{14}$ & 0.2152 & 0.1316 & Verbenene \\
\hline 136.124652 & 136.124654 & 0.0110 & 3 & $\mathrm{C}_{10} \mathrm{H}_{16}$ & 0.3829 & 0.1208 & Pinene \\
\hline 138.103916 & 138.103922 & 0.0434 & 3 & $\mathrm{C}_{9} \mathrm{H}_{14} \mathrm{O}_{1}$ & N.D. & 0.0137 & Sabina ketone \\
\hline 148.088266 & 148.088252 & 0.0945 & 5 & $\mathrm{C}_{10} \mathrm{H}_{12} \mathrm{O}_{1}$ & 0.0423 & 0.0242 & Methyl chavicol \\
\hline 150.103916 & 150.103912 & 0.0266 & 4 & $\mathrm{C}_{10} \mathrm{H}_{14} \mathrm{O}_{1}$ & 0.0469 & 0.0363 & Verbenone \\
\hline 152.119567 & 152.119559 & 0.0559 & 3 & $\mathrm{C}_{10} \mathrm{H}_{16} \mathrm{O}_{1}$ & 0.0235 & 0.0244 & Methyl thymol, Thujone, Piperitone, \\
\hline 162.067531 & 162.067537 & 0.0370 & 6 & $\mathrm{C}_{10} \mathrm{H}_{10} \mathrm{O}_{2}$ & 0.0239 & 0.0137 & Safrole \\
\hline 164.083181 & 164.083170 & 0.0701 & 5 & $\mathrm{C}_{10} \mathrm{H}_{12} \mathrm{O}_{2}$ & 0.0280 & 0.0152 & 2-Phenylethyl acetate \\
\hline 164.119567 & 164.119580 & 0.0792 & 4 & $\mathrm{C}_{11} \mathrm{H}_{16} \mathrm{O}_{1}$ & 0.0387 & 0.0251 & Thymol methyl ether \\
\hline 166.098831 & 166.098834 & 0.0151 & 4 & $\mathrm{C}_{10} \mathrm{H}_{14} \mathrm{O}_{2}$ & 0.0940 & 0.0675 & Perillic acid \\
\hline 168.114481 & 168.114484 & 0.0149 & 3 & $\mathrm{C}_{10} \mathrm{H}_{16} \mathrm{O}_{2}$ & 0.0625 & 0.0465 & Geranic acid \\
\hline 178.098831 & 178.098832 & 0.0028 & 5 & $\mathrm{C}_{11} \mathrm{H}_{14} \mathrm{O}_{2}$ & 0.0440 & 0.0257 & Methyl eugenol \\
\hline 180.078096 & 180.078100 & 0.0222 & 5 & $\mathrm{C}_{10} \mathrm{H}_{12} \mathrm{O}_{3}$ & 0.0362 & 0.0272 & Coniferyl alcohol \\
\hline 182.130131 & 182.130140 & 0.0467 & 3 & $\mathrm{C}_{11} \mathrm{H}_{18} \mathrm{O}_{2}$ & 0.0474 & 0.0360 & Geranyl formate \\
\hline 192.114481 & 192.114489 & 0.0390 & 5 & $\mathrm{C}_{12} \mathrm{H}_{16} \mathrm{O}_{2}$ & 0.0413 & 0.0300 & $\beta$-Phenylethyl isobutyrate \\
\hline 200.155952 & 200.155948 & 0.0200 & 6 & $\mathrm{C}_{15} \mathrm{H}_{20}$ & 0.0973 & 0.0687 & Calorene \\
\hline 202.171602 & 202.171604 & 0.0099 & 5 & $\mathrm{C}_{15} \mathrm{H}_{22}$ & 0.2410 & 0.1885 & Calamenene \\
\hline 204.150867 & 204.150861 & 0.0294 & 5 & $\mathrm{C}_{14} \mathrm{H}_{20} \mathrm{O}_{1}$ & 0.0846 & 0.0549 & Khusimone \\
\hline 204.187252 & 204.187263 & 0.0514 & 4 & $\mathrm{C}_{15} \mathrm{H}_{24}$ & 3.9589 & 3.0658 & Germacrene D, Caryophyllene \\
\hline 206.130131 & 206.130141 & 0.0485 & 5 & $\mathrm{C}_{13} \mathrm{H}_{18} \mathrm{O}_{2}$ & 0.1365 & 0.1051 & Phenylethyl isovalerate \\
\hline 206.166517 & 206.166508 & 0.0437 & 4 & $\mathrm{C}_{14} \mathrm{H}_{22} \mathrm{O}_{1}$ & 0.1094 & 0.0560 & Alpha irone \\
\hline 208.109396 & 208.109415 & 0.0913 & 5 & $\mathrm{C}_{12} \mathrm{H}_{16} \mathrm{O}_{3}$ & N.D. & 0.0452 & Pinchotene acetate \\
\hline 216.150867 & 216.150872 & 0.0231 & 6 & $\mathrm{C}_{15} \mathrm{H}_{20} \mathrm{O}_{1}$ & 0.1767 & 0.1206 & Hexyl cinnamaldehyde \\
\hline 218.166517 & 218.166504 & 0.0596 & 5 & $\mathrm{C}_{15} \mathrm{H}_{22} \mathrm{O}_{1}$ & 0.0790 & 0.0487 & Nootkatone \\
\hline 220.145781 & 220.145782 & 0.0045 & 5 & $\mathrm{C}_{14} \mathrm{H}_{20} \mathrm{O}_{2}$ & 0.2837 & 0.2432 & Phenylethyl hexanoate \\
\hline 220.182167 & 220.182167 & 0.0000 & 4 & $\mathrm{C}_{15} \mathrm{H}_{24} \mathrm{O}_{1}$ & 0.1338 & 0.0899 & Caryophlene oxide, Farnasal, Spathulenol \\
\hline 222.067531 & 222.067501 & 0.1373 & 6 & $\mathrm{C}_{15} \mathrm{H}_{10} \mathrm{O}_{2}$ & 0.0367 & 0.0268 & Aurone, Flavone \\
\hline 222.197817 & 222.197810 & 0.0315 & 3 & $\mathrm{C}_{15} \mathrm{H}_{26} \mathrm{O}_{1}$ & 0.1482 & 0.0515 & Germacrene D-4-ol \\
\hline 224.177081 & 224.177137 & 0.2498 & 3 & $\mathrm{C}_{14} \mathrm{H}_{24} \mathrm{O}_{2}$ & 0.0249 & 0.0000 & Bornyl isobutyrate \\
\hline 234.161431 & 234.161425 & 0.0256 & 5 & $\mathrm{C}_{15} \mathrm{H}_{22} \mathrm{O}_{2}$ & 0.2303 & 0.1844 & Polygodial \\
\hline 236.177081 & 236.177079 & 0.0106 & 4 & $\mathrm{C}_{15} \mathrm{H}_{24} \mathrm{O}_{2}$ & 0.2438 & 0.2002 & Myrtenyl isovalerate \\
\hline 238.156346 & 238.156319 & 0.1155 & 4 & $\mathrm{C}_{14} \mathrm{H}_{22} \mathrm{O}_{3}$ & 0.0226 & 0.0135 & Pinchotene acetate \\
\hline 254.202902 & 254.202904 & 0.0079 & 7 & $\mathrm{C}_{19} \mathrm{H}_{26}$ & 1.1065 & 0.9565 & Norabieta-4(18),8,11,13-tetraene \\
\hline
\end{tabular}




\begin{tabular}{|c|c|c|c|c|c|c|c|}
\hline 256.218552 & 256.218563 & 0.0429 & 6 & $\mathrm{C}_{19} \mathrm{H}_{28}$ & 0.4677 & 1.7426 & Norabietatriene \\
\hline 270.234202 & 270.234212 & 0.0370 & 6 & $\mathrm{C}_{20} \mathrm{H}_{30}$ & 0.1482 & 0.1128 & Abieta-8,11,13-triene \\
\hline 272.249852 & 272.249865 & 0.0478 & 5 & $\mathrm{C}_{20} \mathrm{H}_{32}$ & 5.3297 & 4.0430 & Abietadiene \\
\hline 284.213467 & 284.213473 & 0.0194 & 7 & $\mathrm{C}_{20} \mathrm{H}_{28} \mathrm{O}_{1}$ & 0.6454 & 0.5597 & Dehydroabietal \\
\hline 286.229117 & 286.229121 & 0.0122 & 6 & $\mathrm{C}_{20} \mathrm{H}_{30} \mathrm{O}_{1}$ & 0.5142 & 0.3811 & Ferruginol \\
\hline 288.244767 & 288.244751 & 0.0572 & 5 & $\mathrm{C}_{20} \mathrm{H}_{32} \mathrm{O}_{1}$ & 0.4556 & 0.3226 & Isopimarol \\
\hline 290.260417 & 290.260411 & 0.0207 & 4 & $\mathrm{C}_{20} \mathrm{H}_{34} \mathrm{O}_{1}$ & 2.2275 & 1.6607 & Isoabienol \\
\hline 292.239682 & 292.239679 & 0.0120 & 4 & $\mathrm{C}_{19} \mathrm{H}_{32} \mathrm{O}_{2}$ & 0.4933 & 0.5583 & 15-Norpinifolic acid \\
\hline 300.171996 & 300.171965 & 0.1033 & 8 & $\mathrm{C}_{19} \mathrm{H}_{24} \mathrm{O}_{3}$ & 0.1481 & 0.0998 & Z-Bornyl-p-coumarate \\
\hline 300.208382 & 300.208370 & 0.0416 & 7 & $\mathrm{C}_{20} \mathrm{H}_{28} \mathrm{O}_{2}$ & 4.8995 & 5.5528 & Dehydroabietic acid \\
\hline 302.224032 & 302.223991 & 0.1373 & 6 & $\mathrm{C}_{20} \mathrm{H}_{30} \mathrm{O}_{2}$ & 40.8471 & 45.5916 & Abietic acid \\
\hline 304.239682 & 304.239697 & 0.0493 & 5 & $\mathrm{C}_{20} \mathrm{H}_{32} \mathrm{O}_{2}$ & 1.3418 & 1.0819 & Anticopalic acid \\
\hline 314.187646 & 314.187635 & 0.0366 & 8 & $\mathrm{C}_{20} \mathrm{H}_{26} \mathrm{O}_{3}$ & 0.2962 & 0.2178 & 7-Oxodehydroabietic acid \\
\hline 314.224032 & 314.224027 & 0.0159 & 7 & $\mathrm{C}_{21} \mathrm{H}_{30} \mathrm{O}_{2}$ & 2.6948 & 2.3663 & Methyl dehydroabietate \\
\hline 316.203296 & 316.203295 & 0.0032 & 7 & $\mathrm{C}_{20} \mathrm{H}_{28} \mathrm{O}_{3}$ & 3.0338 & 2.0948 & Lambertianic acid \\
\hline 316.239682 & 316.239666 & 0.0522 & 6 & $\mathrm{C}_{21} \mathrm{H}_{32} \mathrm{O}_{2}$ & 0.2248 & 0.2072 & Methyl sandaracopimarate \\
\hline 318.218946 & 318.218948 & 0.0047 & 6 & $\mathrm{C}_{20} \mathrm{H}_{30} \mathrm{O}_{3}$ & 2.6830 & 2.6527 & 3-Oxoanticopalic acid \\
\hline 318.255332 & 318.255347 & 0.0471 & 5 & $\mathrm{C}_{21} \mathrm{H}_{34} \mathrm{O}_{2}$ & 0.0560 & 0.0827 & Methyl arachidonate \\
\hline 320.234596 & 320.234612 & 0.0500 & 5 & $\mathrm{C}_{20} \mathrm{H}_{32} \mathrm{O}_{3}$ & 1.6867 & 1.5772 & Isocupressic acid \\
\hline 322.250246 & 322.250263 & 0.0528 & 4 & $\mathrm{C}_{20} \mathrm{H}_{34} \mathrm{O}_{3}$ & 0.0688 & 0.0504 & Imbricatolic acid (dihydroisocupressic acid) \\
\hline 330.218946 & 330.218919 & 0.0818 & 7 & $\mathrm{C}_{21} \mathrm{H}_{30} \mathrm{O}_{3}$ & 0.1521 & 0.1060 & Methyl daniellate \\
\hline 332.198211 & 332.198188 & 0.0707 & 7 & $\mathrm{C}_{20} \mathrm{H}_{28} \mathrm{O}_{4}$ & 0.3339 & 0.2482 & Pinusolic acid \\
\hline 332.234596 & 332.234598 & 0.0045 & 6 & $\mathrm{C}_{21} \mathrm{H}_{32} \mathrm{O}_{3}$ & 5.5126 & 5.9585 & $\begin{array}{l}\text { 12-Methoxy-6} 6,11 \text {-dihydroxyabieta- } \\
8,11,13 \text {-triene }\end{array}$ \\
\hline 334.213861 & 334.213891 & 0.0883 & 6 & $\mathrm{C}_{20} \mathrm{H}_{30} \mathrm{O}_{4}$ & 2.6595 & 1.7846 & Dehydropinifolic acid \\
\hline 336.229511 & 336.229518 & 0.0193 & 5 & $\mathrm{C}_{20} \mathrm{H}_{32} \mathrm{O}_{4}$ & 1.4024 & 1.7652 & Pinifolic acid \\
\hline 346.213861 & 346.213891 & 0.1126 & 7 & $\mathrm{C}_{21} \mathrm{H}_{30} \mathrm{O}_{4}$ & 0.1044 & 0.0957 & Pinusolide \\
\hline 350.208775 & 350.208760 & 0.0428 & 6 & $\mathrm{C}_{20} \mathrm{H}_{30} \mathrm{O}_{5}$ & 0.0554 & 0.0499 & Andrographolide \\
\hline 350.245161 & 350.245133 & 0.0785 & 5 & $\mathrm{C}_{21} \mathrm{H}_{34} \mathrm{O}_{4}$ & 12.8697 & 12.5939 & Methyl pinifolate \\
\hline 362.245161 & 362.245159 & 0.0055 & 6 & $\mathrm{C}_{22} \mathrm{H}_{34} \mathrm{O}_{4}$ & 0.2316 & 0.2039 & Acetylisocupressic acid \\
\hline 414.385618 & 414.385526 & 0.2232 & 5 & $\mathrm{C}_{29} \mathrm{H}_{50} \mathrm{O}_{1}$ & 0.0558 & 0.0372 & $\beta$-Sitosterol \\
\hline 430.380532 & 430.380463 & 0.1615 & 5 & $\mathrm{C}_{29} \mathrm{H}_{50} \mathrm{O}_{2}$ & 0.0555 & 0.0181 & Tocopherol \\
\hline 446.339061 & 446.339044 & 0.0381 & 6 & $\mathrm{C}_{28} \mathrm{H}_{46} \mathrm{O}_{4}$ & 0.0422 & 0.0249 & Didecyl phthalate \\
\hline
\end{tabular}

N.D. - Not detected 


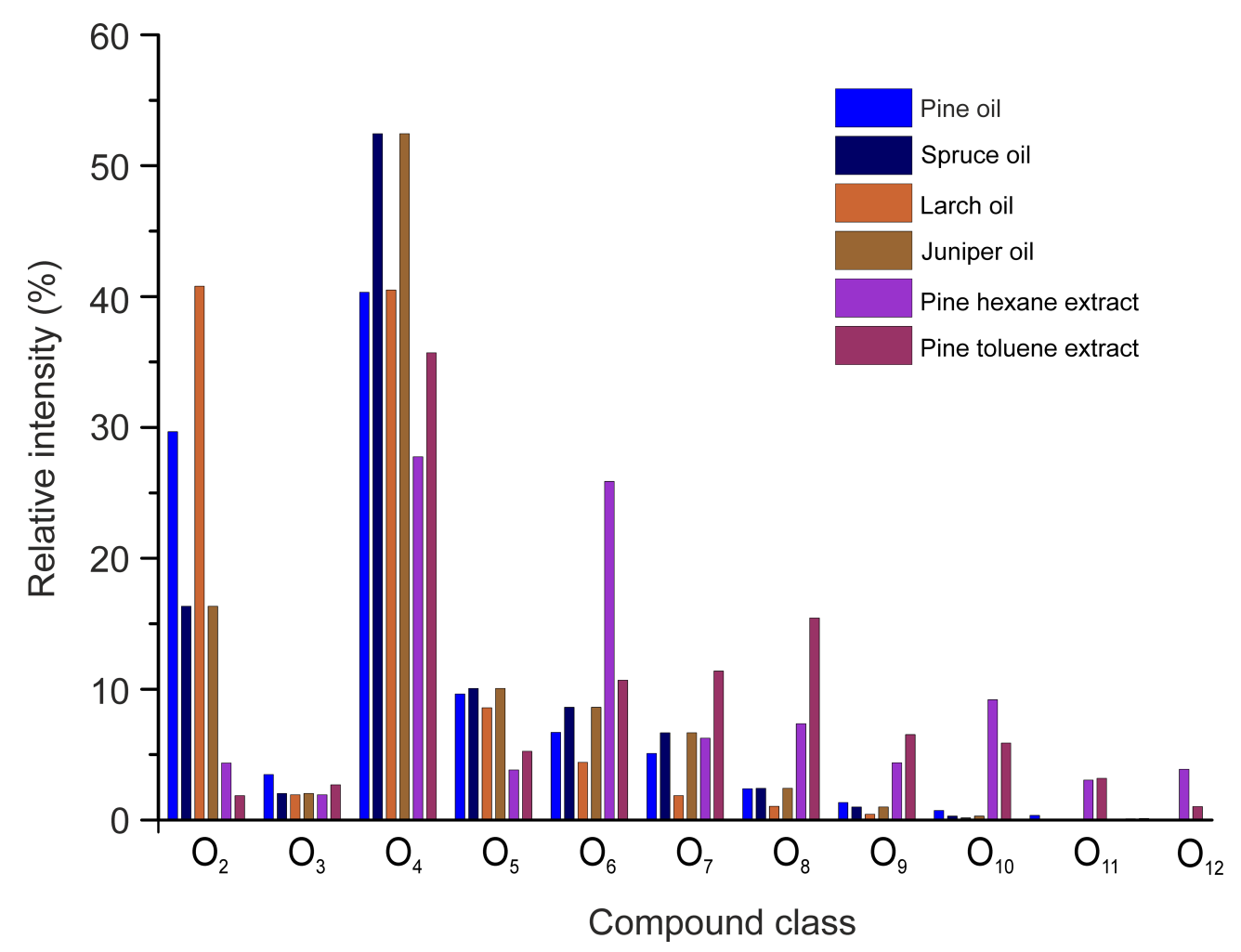

Figure S1. Negative-ion ESI compound class distributions of conifer needle essential oils and solvent extracts.

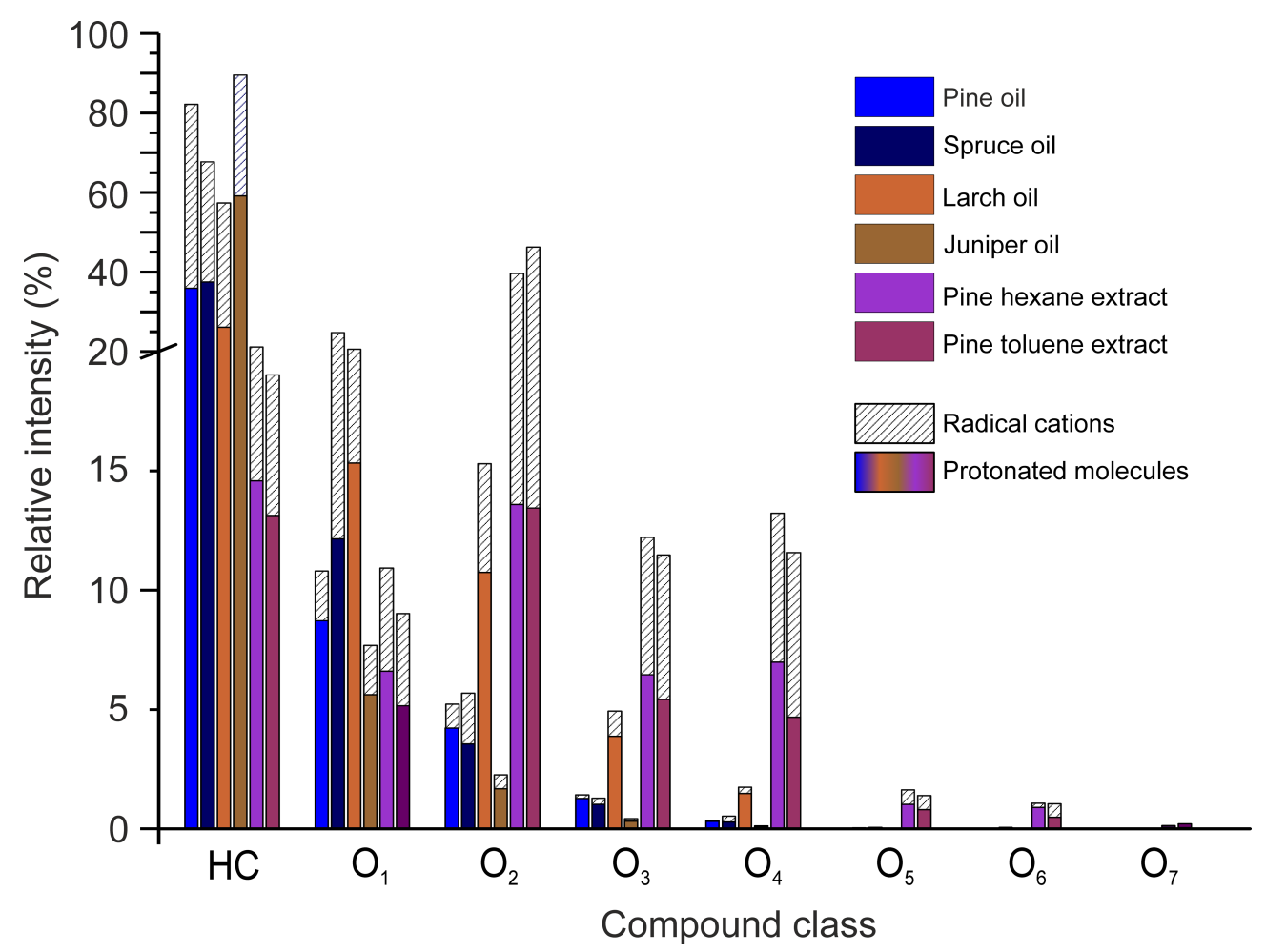

Figure S2. Positive-ion APPI compound class distributions of conifer needle essential oils and solvent extracts. 